\title{
Characterisation, in-vitro and in-vivo evaluation of valproic acid-loaded nanoemulsion for improved brain bioavailability
}

\begin{abstract}
OBJECTIVE: This study was aimed to investigate the potential of formulated valproic acidencapsulated nanoemulsion (VANE) to improve the brain bioavailability of valproic acid (VPA). METHODS: Valproic acid-encapsulated nanoemulsions were formulated and physically characterised (osmolarity, viscosity, drug content, drug encapsulation efficiency). Further investigations were also conducted to estimate the drug release, cytotoxic profile, invitro blood-brain barrier (BBB) permeability, pharmacokinetic parameter and the concentration of VPA and VANE in blood and brain. KEY FINDINGS: Physical characterisation confirmed that VANE was suitable for parenteral administration. Formulating VPA into nanoemulsion significantly reduced the cytotoxicity of VPA. In-vitro drug permeation suggested that VANEs crossed the BBB as freely as VPA. Pharmacokinetic parameters of VANE-treated rats in plasma and brain showed F3 VANE had a remarkable improvement in AUC, prolongation of half-life and reduction in clearance compared to VPA. Given the same extent of in-vitro BBB permeation of VPA and VANE, the higher bioavailability of VANE in brain was believed to have due to higher concentration of VANE in blood. The brain bioavailability of VPA was improved by prolonging the half-life of VPA by encapsulating it within the nanoemulsion-T80. CONCLUSIONS: Nanoemulsion containing VPA has alleviated the cytotoxic effect of VPA and improved the plasma and brain bioavailability for parenteral delivery of VPA.
\end{abstract}

Keyword: Blood-brain barrier; In-vitro model; Parenteral nanoemulsion; Pharmacokinetics; Valproic acid 